\title{
Self-organization Framework for Mobile Ad Hoc Networks
}

\author{
Ali Kies ${ }^{\# 1}$, Sara Mehar ${ }^{* 2}$, Belbachir Rodwane ${ }^{\# 3}$, Zoulikha Mekkakia Maaza ${ }^{\# 4}$, Sidi Mohammed Senouci ${ }^{* 5}$

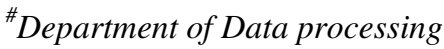 \\ University of Sciences and the Technology of Oran, USTO \\ BP 1505 El M'Naouar, Oran, Algeria \\ 1,3,4 \{kies_ali, balbachir_redouane,mekkakia\}@univ-usto.dz \\ *University of Bourgogne, DRIVE Lab \\ 49 Rue Mademoiselle Bourgeois, 58000, Nevers, France \\ ${ }^{2}$ Sara_Mehar@etu.u-bourgogne.fr, 5idi-Mohammed.Senouci@u-bourgogne.fr
}

\begin{abstract}
Mobile ad hoc networks (MANETs) face a number of challenges, in particular due to dynamic network topology and large variable number of mobile nodes. To overcome these problems an effective solution is to define a self-organizing architecture that facilitates the network management task and permits to deploy wide panoply of services. One method to organize such networks is to define a virtual backbone that covers all the nodes in the network. In this paper we propose new self-organization architecture based on an optimized CDS construction. In this approach, the neighbors select CDS nodes intelligibly. In the selection processes, a new weight parameter depending on energy, link quality and connectivity is introduced in order to increase the CDS lifetime and hence the network performances. To study the benefits of this new architecture, we propose a new routing protocol called "PROC" (Proactive Routing based on Optimized CDS). Simulation results, using NS2, show that PROC minimizes the overhead and the energy consumption compared to the well-known OLSR routing protocol.
\end{abstract}

Keywords: mobile ad hoc networks, routing protocols, data dissemination, self-organization, backbone, CDS.

\section{INTRODUCTION}

A mobile ad hoc network is a network where many devices communicate with one another in an ad hoc fashion over multiple hops, most often wirelessly. Due to their specific characteristics (nodes' mobility, limited resources, etc.), MANETs still present significant technical challenges. In order to build such a network and communicate efficiently between a large-scale of heterogeneous mobile nodes, some form of organization has to take place.

The term "Self-organization" has been introduced in the 60 's in cybernetics and in the 70's in physics of complex systems. It is described as a mechanism through which individual elements in a group interact locally yet give the group a macroscopic property, often described as an organization or a structure.

Self-organizing an ad hoc network allows the creation of a different view of radio topology called virtual structure, introducing one or more hierarchy levels and facilitates the establishment of the services necessary to the expected network operation such as routing [1]. The functions that self-organization should accomplish in such networks are: (i) resource sharing, (ii) structure formation and maintenance, (iii) helping the deployment of communication protocols, and (iv) resource management.

We can classify self-organization from their scope in the network into two categories: backbone-based and clusterbased. In the first category, the protocols aim at generating a virtual backbone that covers all nodes in the network. Some virtual backbones represent a dominating set of the network (in case of $\mathrm{CDS}^{1}$ for example). In the cluster-based category, the nodes are grouped into a set of clusters. Generally in each cluster, a specific node called leader or Cluster-Head $(\mathrm{CH})$ is designed to be in charge of a set of specific functionalities within its cluster [2].

We focus in this work on the first category: backbone based self-organization and especially CDS. A CDS represents a set of nodes such that any node in the network is a neighbor of some element of the set. It is connected if the sub-graph formed by this set is connected. The connected dominating set property ensures that all nodes will receive a broadcast packet. To optimize the broadcast, the node retransmits the packet if it has not already received it and the node is in the connected dominating set. Moreover, the selection of the connected dominating set must be distributed. Based on neighborhood knowledge, a node has to decide whether or not it is in the dominating set. A CDS is a good candidate of a virtual backbone for wireless networks, because any non-CDS node in the network has one-hop distance from a CDS node, which makes routing easier.

Actually, in the classical CDS selection algorithms, each node selects itself as CDS node among its neighbors and broadcasts this information. This scheme increases the overhead. Therefore, we propose a new optimized approach to construct a CDS. In this approach, the CDS node is selected by its neighbors without flooding this information. In the selection process, we introduce a new weight parameter depending on energy, link quality and connectivity to limit disconnection of CDS nodes and hence increase the CDS lifetime.

${ }^{1}$ CDS: Connected Dominating Set. 
We also propose a new routing protocol PROC (Proactive Routing based on Optimized CDS) based on the constructed CDS. PROC optimizes the control packets dissemination and energy consumption in the mobile ad hoc network.

The rest of the paper is organized as follows. Section II presents and discusses some related work on selforganization in MANETs. Section III formulates the problem and describes the self-organization architecture. Section IV details PROC routing protocol. Finally, section V presents simulation results before concluding in section VI.

\section{BACKGROAND AND RELATED WORKS}

The term "Self-organization" has been introduced in the 60 's in cybernetics and in the 70's in physics of complex systems. It is described as a mechanism through which individual elements in a group interact locally yet give the group a macroscopic property, often described as an organization or a structure. The functions that selforganization must accomplish in such networks [3] are:

- Resource sharing: Resources in wireless networks are shared between all nodes in the network (e.g. bandwidth, electromagnetic spectrum, etc.). Self-organization aims to find out how best to (re)allocate these resources, to learn about participants and demands, to supervise changes and to adapt to these changes,

- Structure formation and maintenance: Wireless networks don't have static physical topologies. The goal of self-organization is to form efficient structures and to maintain them. The efficiency can be related to the energy consumption in the case of mobile ad hoc networks or the stability of the structure in the vehicular ad hoc network case,

- Helping the deployment of communication protocols: Self-organization aims to facilitate the deployment of communication protocols such as configuration, routing, dissemination, etc. For configuration, in the absence of a centralized entity, the self-organization protocol should help the allocation of address to all the nodes in the network. Self-organization protocols should also facilitate the routing of data by conserving connectivity of the network,

- Resource management: In wireless networks, selforganization should also manage resources like synchronization, power conservation. For example, the structure which will be constructed by the selforganizing protocol must not only choose nodes having more energy in power constraint networks, but also to balance the energy consumption.

We can classify self-organization into two categories: backbone-based and cluster-based. In the first category, the protocols aim at generating a virtual backbone that covers all nodes in the network. Some virtual backbones represent a dominating set of the network (in case of CDS and MIS), while the others contain all nodes but with a subset of communication links (in case of LMST and RNG). Nevertheless, the goal stays the same: simplifying the physical structure and providing a logic structure based on localized decisions. In [4], the authors propose a localized algorithm to construct a CDS. First step of this algorithm is to mark the nodes having at least two neighbors not directly connected as dominant nodes (become node in the CDS). Then, it applies two dominating reducing rules to reduce some redundant nodes in the CDS. Rule 1: a marked node can unmark itself if another marked node covers it. Rule 2: another marked node can unmark it, if it is covered by two other directly connected marked neighbors. Node $A$ is covered by node $B$ means all $A$ 's neighbor is a subset of $B$ 's neighbors. Each node learns its neighbor information and status (mark or unmark) before next reducing process, through broadcasting Hello messages. So it imposes important communication overhead and energy consumption is high. To reduce communication overhead, [5] extends rules to select/reduce the connected set of dominant nodes, which prolongs the network lifetime. The rules are based on energy and neighborhood degree. Also [6] proposes to improve the algorithm of [4] where nodes are assumed to have a record key (degree, $x, y$ ), where $x$ and $y$ are location coordinates. This record is used to apply rules 1 and 2. In which they define that a node can unmark itself if it is covered by one or two connected neighbors, no matter whether they are marked or not. [6] proposes the creation of a source oriented CDS in order to optimize the diffusion of information. In [7], the authors propose an iterative exploration starting from a leader in the network to allow the building and interconnecting tree. The approach is based on DS (Dominating Set) construction and its interconnection. A node has one of four following states: initial state, dominating (CDS member), dominated (neighbor of a dominant) and active (in election). An active node that has the highest weight among its active neighbors becomes dominant, its neighbors becomes dominated ones. The weight depends on degree value, node identifier and energy level. In [8] they use the concept of multipoint relays MPR. The idea of MPR is to use the knowledge of two-hop to optimize local broadcast. Thus, each node selects a multipoint relays set among its neighbors, allowing it to attain all nodes at two hops. Among the routing protocols based on backbone topology, we find Optimized Link State Routing (OLSR) based on the concept of multipoint relay (MPR) [8].

In the cluster-based category, the nodes are grouped into a set of clusters. Generally in each cluster, a specific node called leader or Cluster-Head $(\mathrm{CH})$ is designed to be in charge of a set of specific functionalities within its cluster. In [9], the node that has the higher identifier among all its neighbors is elected as $\mathrm{CH}$. The $\mathrm{CH}$ and all its neighbors form the cluster. In [10], the election of CHs is based on the degree of connectivity (neighbor's number of node) instead of the identities of the nodes. In [11], the weight is defined as the speed of each node. Assuming that all the nodes are equipped with a GPS, the authors in [12] define the $\mathrm{CH}$ as the node that has the minimal average distance between it and the other nodes of the group. There exists some routing protocols based on this cluster-based category, we cite Cluster Based Routing Protocol (CBRP) [13], zone routing protocol (ZRP) [14] and Clusterpow protocol [15].

As mentioned before, there is also a third category that integrates both. In [2], the authors present a distributed 
algorithm for constructing K-CDS: the distance between a node and the CDS is $\mathrm{K}$ hops. Construction and interconnection of the K-CDS are inspired from [7]. In this paper, the presence of a leader is obligatory. In a hybrid network with an existing Access Point (AP), the AP plays the role of natural leader [2]. If instead there is no existing AP, it is necessary to elect node using the algorithm described in [16]. After the CDS construction phase, a $\mathrm{CH}$ election phase is triggered. Only nodes that are members of CDS do $\mathrm{CH}$ election. In [2], the authors propose Virtual Structure Routing (VSR) based on the previous virtual topology.

The clustered networks have some critical points; especially in the cluster-head election algorithm because this phase introduce a latency in the network. Furthermore, this category of self-organizing networks needs a specific attention when selecting the $\mathrm{CHs}$ because they consume more energy than other nodes, so there is a need to use good metrics for the $\mathrm{CH}$ election. The protocols based on backbone structure aims to simplify the physical structure and to provide a logic structure based on localized decisions. Actually, in the classical CDS selection algorithms, each node selects itself as CDS node among its neighbors and broadcasts this information. This behavior increases the control traffic. Therefore, we propose a new optimized approach to construct a CDS. In this approach, the CDS node is selected by its neighbors without flooding this information. In the selection processes, we introduce a new weight parameter depending on energy, link quality and connectivity to limit disconnection of CDS nodes and hence increase the CDS lifetime.

\section{PROPOSED SELF-ORGANIZATION ARCHITECTURE}

In this section, we present our self-organization structure for mobile ad hoc networks. This virtual structure belongs to the backbone-based category and proposes a new optimized approach to construct the CDS. In this structure, the nodes are divided into dominant nodes (CDS nodes) and dominated nodes (not-CDS nodes). We chose to build our CDS as the form 1-CDS, the distance between a node and CDS is one hop, to limit disconnections in the network. To build our $\mathrm{CDS}$, neighborhood knowledge is required. So, each node periodically broadcasts a hello message. The CDS construction is divided into two phases. Initially, each node maintains a neighborhood table that contains all the information of its neighbors. Based on this table, each node in the network selects intelligibly a node in its neighborhood to be the dominant node (DN), i.e, CDS node. In the selection processes, a new "weight" parameter depending on energy, quality of the link and connectivity is introduced. This will allow increasing the CDS lifetime and hence enhance the performances of the mobile ad hoc network.

We detail in the following how this structure is built.

As mentioned before, each node broadcasts hello messages, containing its weight, and information about its neighbors and their link status. The link status is set to "DN" for the neighbor that was selected as a dominant node. The format of a hello message is illustrated in the following Figure:

\begin{tabular}{|c|c|}
\hline Message Sequence Number & CDS Sequence number \\
\hline Link Type (CDS) & Link Message Size \\
\hline Neighbor Address \\
\hline \multicolumn{2}{|c|}{$\ldots$} \\
\hline Link Type(not CDS) & Link Message Size \\
\hline \multicolumn{2}{|c|}{ Neighbor Address } \\
\hline \multicolumn{2}{|c|}{$\ldots$} \\
\hline
\end{tabular}

Fig. 1 Hello message format.

After receiving a hello message from a neighbor $N$, each node maintains a neighborhood table. As shown in Table 1, each entry in this table contains information about this neighbor $\mathrm{N}$ : id, weight and the link status with this neighbor, and an associated lifetime. The lifetime is the lifetime of the neighbor table entry.

TABLE I

NEIGHBORHOOD TABLE ENTRY

\begin{tabular}{|l|l|l|l|}
\hline sender id & sender weight & link status & lifetime \\
\hline
\end{tabular}

Using this table, each node in the network selects a node in its neighborhood that has the maximum weight to be the dominant node. Each dominated node constructs also a CDS table. In this table, the CDS node registers only the addresses of the one-hop neighbors that select him as dominant node. The dominated nodes will hence have an empty CDS table. Each entry of the CDS table will have the following format shown in Table 2.

TABLE II

CDS TABLE ENTRY

node id $\quad$ lifetime

As illustrated in Fig. 2, each CDS node has the maximum weight. For example, node 9 has five neighbors: node 17 with a weight of 3.61 , node 14 with a weight of 3.50 , node 8 with a weight of 2.10 , node 6 with a weight of 2.11 , and node 7 with a weight of 2.41 . Hence, node 9 will choose node 17 as a dominant node.

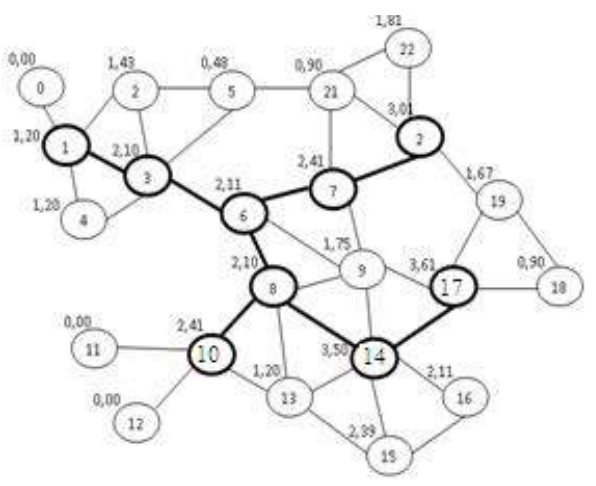

Fig. 2 CDS construction.

The dominant node of node $n$ is denoted $D N(n)$. Each node maintains information about its DN. The DN is recomputed when a change in the weight of neighborhood is detected or a new neighbor with a higher weight appeared. Nodes obtain this information from the periodic hello messages received from their neighbors. 
For the CDS construction process, we define these two well-known following properties for each node:

Propriety 1: each dominated nodes is adjacent to a dominant node:

$$
\forall \mathrm{u} \in \mathrm{V}, \exists \mathrm{v} \in \mathrm{V}^{-} / \mathrm{v} \in \mathrm{N}(\mathrm{u})
$$

Propriety 2: dominant nodes are all connected:

$$
\forall \mathrm{u} \in \mathrm{V}^{-}, \exists \mathrm{v} \in \mathrm{V}^{-} / \mathrm{v} \in \mathrm{N}(\mathrm{u})
$$

Where, $V$ represents all the nodes and $V$ ' represents CDS nodes, and $N(u)$ the neighbors of node $u$.

It is obvious that the choice of a bad dominant node will lead to a significant decrease of the network performance. In fact, the choice of a dominant with an energy level too low will introduce a rupture and disconnection in the structure as the selection of a node with a low connectivity degree. Hence, we introduce this new "weight" parameter that depends on energy, quality of the link and connectivity. The weight is defined as follows:

$$
P \text { selection }=\propto . D+\beta \cdot E+\gamma \cdot M
$$

Where:

$D$ : represents the degree of a node

$E$ : is the remaining energy level

$M$ : is the evaluation of the received signal strength

$\alpha, \beta$ and $\gamma$ : are weighting factors depending on whether we want to focus on nodes with a strong neighborhood or high energy or good quality of the signal.

\section{PROC ROUTING PROTOCOL}

In this section, we present our proactive routing protocol based on the proposed optimized CDS structure (PROC, Proactive Routing based on Optimized CDS). In order to build routes to all nodes in the network, each dominant node broadcasts specific messages called Interconnection Message (IM). IM messages are forwarded, to all nodes of the network through the CDS nodes. An IM message is sent by a dominant node in the network to declare its CDS table, i.e, the addresses of those one-hop neighbor nodes that have

\begin{tabular}{|c|c|}
\hline Message Sequence Number & CDS Selector Sequence Number \\
\hline \multicolumn{2}{|c|}{ Hop Count } \\
\hline \multicolumn{2}{|c|}{ Source Address (Dominant node) } \\
\hline Neighbor $i$ that was $s$ & ected as a dominant node \\
\hline
\end{tabular}
selected him as a DN. The format of an IM message is illustrated in the following Figure. 3:

$$
\text { Fig. } 3 \text { IM message format. }
$$

This helps each node to compute its topology table. Topology table gives a global overview of the virtual CDS structure. Note that only CDS nodes are allowed to rebroadcast the IM messages, which will reduce significantly the number of rebroadcasts in the network. So, each node in the network maintains this topology table, in which it records the information about the virtual topology of the network (see Table 3).
TABLE III

TOPOLOGY TABLE ENTRY

\begin{tabular}{|l|l|l|}
\hline Node id & Dominant Node id & lifetime \\
\hline
\end{tabular}

The "Dominant Node id" is a dominant node of "Node id" node. Each topology entry has an associated lifetime. Upon its expiration, the entry becomes no longer valid and hence must be removed. Note also that when a change in the CDS table is detected, an IM message should be transmitted.

In the following, we introduce a new algorithm that computes the routing table in each node based on local information of neighborhood table and topology table. This routing table is calculated and updated using the following algorithm:

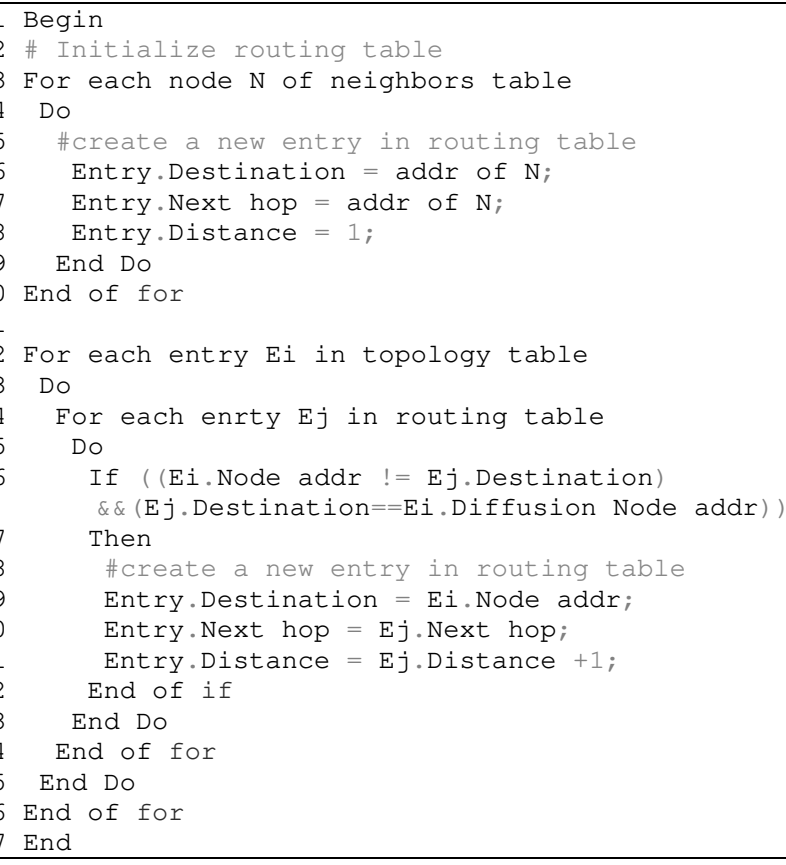

\section{Simulation Results}

We conducted extensive simulations, using NS2 [17], to determine the effectiveness of our routing protocol PROC and compared to the well-known routing protocol OLSR [8]. OLSR and PROC use hello messages to construct the neighborhood table. From these messages, OLSR constructs the MPR nodes and PROC constructs the CDS nodes. OLSR broadcasts the Topology Control messages (TC) and PROC disseminates the IM messages to build the virtual global view of the networks. In Table 4, we define the time interval for sending each message.

TABLE IV

SENDING MESSAGES TIME INTERVALS

\begin{tabular}{|c|c|c|}
\hline OLSR & PROC & Interval \\
\hline Hello message & Hello message & 2 seconds \\
\hline TC & IM & 5 seconds \\
\hline
\end{tabular}

Nodes move according to the Random Waypoint mobility Model (RWP). In RWP mobility model the nodes move from one waypoint to the next. A specific speed and duration is 
chosen for every transition. After the stipulated transition duration ends the node may pause for a specific duration of time before starting its transition towards the next waypoint. Table 5, gives the simulation parameters.

TABLE V

SIMULATION PARAMETERS

\begin{tabular}{|c|c|}
\hline Parameter & Value \\
\hline Number of nodes & $20-100$ \\
\hline Simulation time & $1000 \mathrm{~s}$ \\
\hline Radio range & $250 \mathrm{~m}$ \\
\hline Velocity & $2-10 \mathrm{~m} / \mathrm{s}$ \\
\hline Simulation area & $900 \times 900 \mathrm{~m}^{2}$ \\
\hline$\alpha$ & 0.4 \\
\hline $\mathrm{B}$ & 0.5 \\
\hline$\gamma$ & 0.1 \\
\hline MAC & IEEE $802.11 \mathrm{~b}$ \\
\hline Initial energy & 1000 Joule \\
\hline Tx power, Rx power, Idle power & $1.4,1.2,0.9$ (Watt) \\
\hline
\end{tabular}

To evaluate the performances of our protocol, we focused on two performance metrics: overhead and consumed energy while considering mobility and density. We set $\alpha$ to $0.4, \beta$ to 0.5 and $\gamma$ to 0.1 . This choice implies that we give the priority for the nodes that have the best connectivity, then the nodes with more energy and at the end nodes with a good link quality. Our initial tests have shown that these values give the best results. For each scenario, we have ten random simulations. Then we calculate the average of overhead and energy consumption.

In Fig. 4, we plot the overhead depending on network density. We notice that the overhead generated by PROC is lower than the overhead generated by OLSR. By cons, the difference of overhead between the two protocols is about $11.94 * 10^{4}$ and $109,56 * 10^{4}$ bytes when the nodes' density is 40 to and 100 respectively. So the behavior of our protocol is better when increasing the nodes number. This behavior is due to the optimization of CDS construction.

We also show in Fig. 5 that the average consumed energy is lower in our protocol. This allows enhancing network lifetime. Actually, the introduced weight metric (equation 3) is very important to select the best nodes as dominant nodes in order to avoid the frequent topology update.

To evaluate the effect of the mobility, we used different scenarios by varying the nodes' velocity. We chose a RWP mobility model with a pause time of $4 \mathrm{~s}$ and a speed varying from $2 \mathrm{~m} / \mathrm{s}$ to $10 \mathrm{~m} / \mathrm{s}$.

Fig. 6 shows that the generated overhead and energy consumption are slightly affected by mobility. In fact, mobility induces some topological changes, which induces an increase of the number of update messages. When the virtual topology changes in OLSR, it broadcasts control messages to the entire network, which happens frequently. In PROC, we broadcast control messages only when a node with a higher weight is detected. We can notice that our protocol has better performances compared to OLSR, which means that the CDS structure dynamically adapts to the changes in the environment.

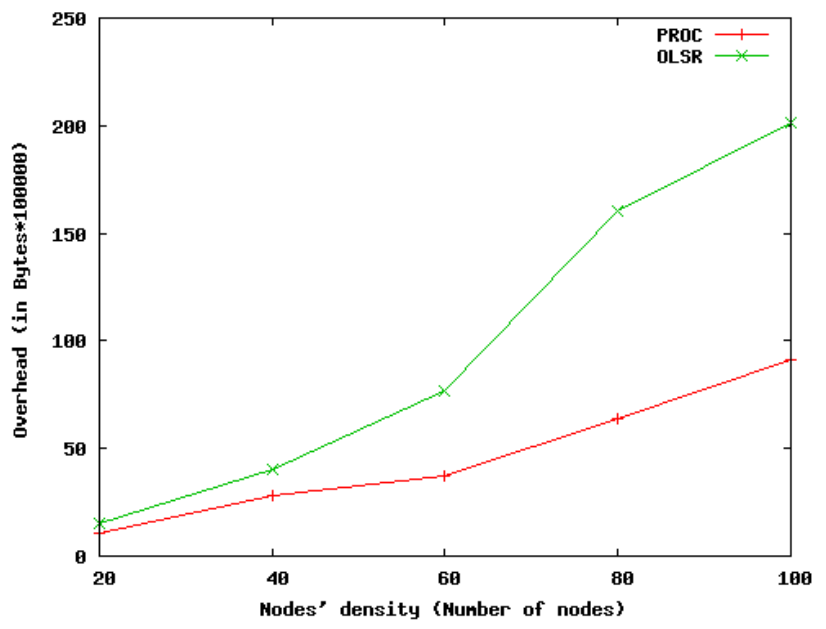

Fig. 4 Overhead vs. nodes' density.

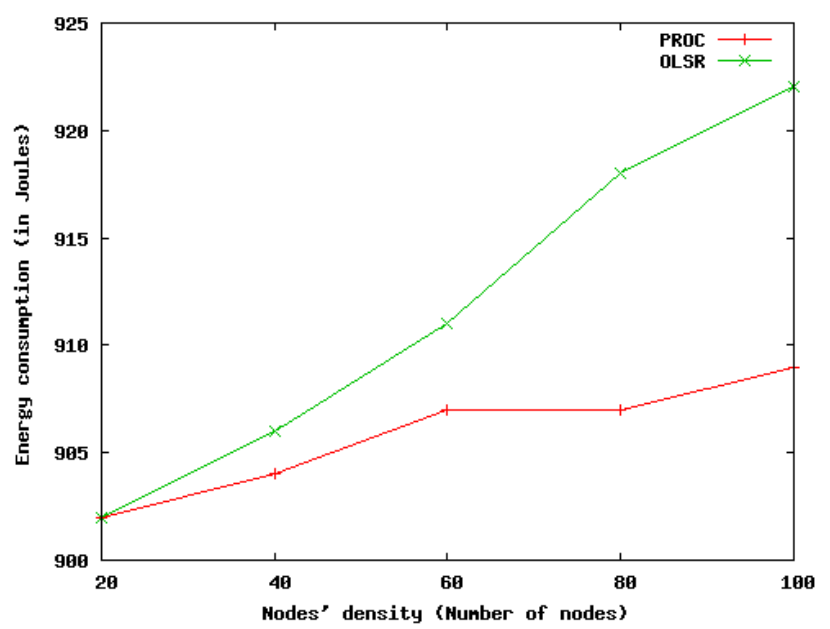

Fig. 5 Energy consumption vs. nodes' density.

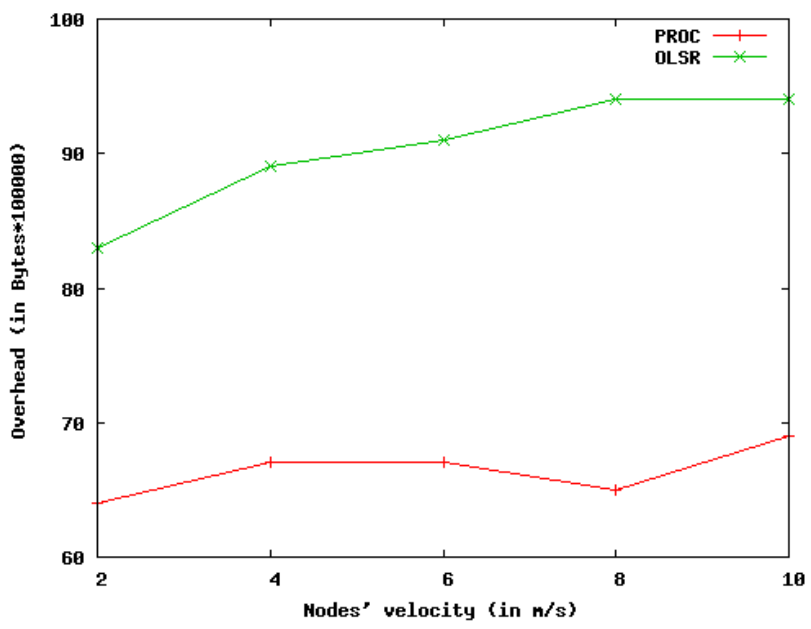

Fig. 6 Overhead vs. nodes' mobility

To improve the survivability of the network, we must use strong nodes in terms of weight, reduce energy consumption and distribute the load. Fig. 7 gives the energy consumption of each node in a network of 60 nodes with a mobility of 8 
$\mathrm{m} / \mathrm{s}$ and a simulation time of 1000 seconds. We present the results of PROC compared to OLSR.

We can notice from Fig. 7, that the energy consumption in PROC is less than OLSR. In fact, the exchange of control packets generated by OLSR is higher than PROC as illustrated in Fig. 6. In OLSR, MPRs set changes if there are changes in two hops neighborhood. This implies a permanent generation of TC message in the network to maintain routing tables. In PROC, CDS nodes are selected to be as stable as possible to limit disconnections. Our algorithm tends to select a node that has a maximum energy to maximize the CDS lifetime, and the best connectivity and signal strength to maintain the CDS connectivity. Border nodes do not participate in network management because the flooding of control messages will be useless. For this, we notice that some nodes consume less than others. Also, we find that the energy consumption is distributed between the nodes using PROC, because if there is degradation of the battery level to a node in the CDS, an automatic re-election of CDS nodes result according to our algorithm (use equation (3)).

If the network is static, MPRs set does not change which means a significant degradation of battery level in MPR node. By cons, in PROC re-election is done according the weight of the nodes to distribute energy consumption to all network nodes. We find that our protocol PROC reacts well with or without mobility.

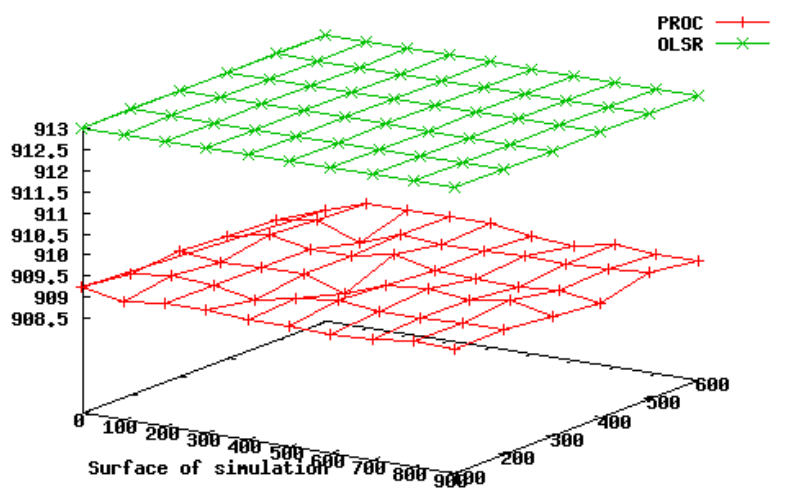

Fig. 7 Energy consumption of all nodes in the network

\section{CONCLUSION}

In this paper, we proposed a new self-organizing framework for mobile ad hoc networks based on an optimized CDS construction. Such framework aims to facilitate the network management task and permits to deploy wide panoply of services and protocols. The CDS construction is done intelligibly since the dominant node selection process uses a new weight parameter depending on energy, quality of the link and connectivity. This parameter is introduced in order to increase the CDS lifetime and hence enhance the network performances.

To demonstrate the effectiveness of our virtual structure, we proposed and implemented a new a routing protocol called PROC based on it. Simulation results show that PROC optimizes the energy consumption within the network and also has the advantage of supporting mobility and minimizes the overhead.

We are currently extending this work by performing other extensive simulations to compare our routing protocol with other existing routing protocols.

\section{REFERENCES}

[1] S. K. Sarkar, T. G. Basavaraju, C. Puttamadappa, "Ad Hoc Mobile Wireless Networks, Principles, Protocoles, and Application”, Taylor edition, 2008.

[2] F.Theoleyre and F.Valois, "Virtual structure routing in ad hoc networks", IEEE ICC'2005, Seoul, Korea, May 2005.

[3] K.L.Mills, "A brief survey of self-organization in wireless sensor networks", Wireless Communications and Mobile Computing, Volume 7, September 2007.

[4] J.Wu and H.Li, "On calculating connected dominating set for efficient routing in ad hoc wireless networks", the 3rd Int'l Workshop on Discrete Algorithms and Methods for Mobile Computing and Communication (DIAL'M), Seattle, USA, August 1999.

[5] H.Li and J.Wu., "Dominating-set-based routing in ad hoc wireless networks", In International Workshop on Discrete Algorithms and Methods for Mobile Computing and Communications(DIAL'M), Seattle, USA, August 1999.

[6] I.Stojmenovic, M.Seddigh and J.Zunic, "Dominating sets and neighbor elimination based broadcasting algorithms in wireless networks", IEEE Transactions on Parallel and Distributed Systems, Volume 15, November 2004.

[7] X.Cheng and D.Du, "Virtual backbone-based routing in multihop ad hoc wireless networks", Technical Report 02-002, University of Minnesota, Minnesota, USA, January 2002.

[8] T. Clausen and P. Jacquet, "Optimized Link State Routing Protocol (OLSR)", RFC 3626, IETF Network Working Group, http://www.ietf.org/rfc/rfc3626.txt, October 2003.

[9] B.Haggar, "Self-Stabilizing Clustering Algorithm for Ad Hoc Networks", The Fifth International Conference on Wireless and Mobile Communications, ICWMC 2009, French Riviera, France, August 2009.

[10] H. Taniguchi, M. Inoue, T. Masuzawa and H. fujiwara, "Clustering Algorithms In Ad Hoc Networks", Electronics and Communications, Volume 88, Japan, January 2005.

[11] S. Basagni, "Distributed Clustering Ad Hoc Networks", In Proceedings of the IEEE International Symposium on Parallel Architectures, Algorithms, and Networks (I-SPAN), Fremantle, Australia, Juin 1999.

[12] N. Benaouda, H.Guyennet, A.Hammad and M. Mostefai, " A New Two Level Hierarchy Structuring for node Partitionning in Ad Hoc Networks", In SAC'10, 25th ACM Symposium on Applied Computing, Zurich, Switzerland, March 2010.

[13] M.Jiang, J.Li and Y.C.Tay, "Cluster Based Routing Protocol(CBRP)", Internet Draft, draft-ietf-manet-cbrp-spec-01.txt, August 1999.

[14] J. Haas and R. Pearlman, "The Zone Routing Protocol (ZRP) for Ad Hoc Networks", Internet Draft, draft-haas-zone-routing-protocol00.txt, November 1997.

[15] P.Santi, " Topology Control in Wireless Ad Hoc and Sensor Networks", Wiley edition, 2005.

[16] N.Malpani, J. L.Welch and N. H. Vaidya, "Leader Election Algorithms for Mobile Ad Hoc Networks", Fourth International Workshop on Discrete Algorithms and Methods for Mobile Computing and Communications, Boston, USA, August 2000.

[17] Network Simulator NS2, http://www.isi.edu/nsnam/ns/index.html. 